\title{
OTIMIZAÇÃO DA EXTRAÇÃO SÓLIDO-LÍQUIDO COM PARTIÇÃO EM BAIXA TEMPERATURA PARA DETERMINAÇÃO DE CARBOFURANO EM Cucurbita pepo L ("ABOBRINHA") POR CROMATOGRAFIA LÍQUIDA DE ALTA EFICIÊNCIA
}

\author{
Rogério P. Rodrigues ${ }^{\mathrm{a}}$, Waldiclécio R. Farias ${ }^{\mathrm{a}}$, Simone M. Goularta,*, Adilson C. Goulart ${ }^{\mathrm{b}}$, João Paulo V. Santos ${ }^{\mathrm{a}}$ e Maria \\ Eliana L. R. de Queiroz \\ aDepartamento de Química, Instituto Federal de Goiás, Av. Furnas, 55, 75524-010 Itumbiara - GO, Brasil \\ ${ }^{b}$ Departamento de Agronomia, Universidade Federal de Uberlândia, Av. Amazonas, s/n, 38400-902 Uberlândia - MG, Brasil \\ 'Departamento de Química, Universidade Federal de Viçosa, Vila Gianetti, Casa 24, 36570-000 Viçosa - MG, Brasil
}

Recebido em 28/06/2017; aceito em 04/09/2017; publicado na web em 27/10/2017

\begin{abstract}
OPTIMIZATION OF SOLID-LIQUID EXTRACTION WITH LOW TEMPERATURE PARTITION FOR DETERMINATION OF CARBOFURAN IN CUCURBITA PEPO L (“ZUCCHINI") BY HIGH PERFORMANCE LIQUID CHROMATOGRAPHY. According to results of the Program of Analysis of Pesticide Residues in Foods of the National Agency of Sanitary in Brazil (2013 to 2015) the zucchini, Cucurbita pepo L is one of the non-hardy vegetables, that presented higher contamination index by pesticides residues. One of these agrochemicals is carbofuran, which although not authorized for this crop, is commonly and illegally used for whitefly control. The objective of this study was to optimize the solid-liquid extraction method with low temperature partitioning (SLE/LTP) for the determination of carbofuran residues in zucchini by high performance liquid chromatography with ultraviolet detector (HPLC-UV). The optimization was performed with a complete factorial design, $2^{3}$ to evaluate the simultaneous performance of three factors in two levels, in triplicate. The optimized SLE/LTP-HPLC/UV method showed to be selective, simple and efficient, consuming a small amount of sample and solvent extractor. The average recovery percentage was $75.88 \%$. Samples of zucchini purchased in the Itumbiara-GO trade, Brazil, and submitted to the optimized method did not present carbofuran residues.
\end{abstract}

Keywords: carbofuran; zucchini; SLE/LTP.

\section{INTRODUÇÃo}

Durante as últimas décadas, o monitoramento de resíduos de agrotóxicos em alimentos no Brasil foi marcado por uma série de esforços isolados de órgãos estaduais de saúde, agricultura e instituições de pesquisas. Esse fato sempre impediu que o país tivesse uma noção clara dos níveis de agrotóxicos encontrados em seus produtos agrícolas. Em 2001, com o intuito de resolver esse tipo de problema, foi criado pela Agência Nacional de Vigilância Sanitária (ANVISA) o Programa de Análises de Resíduos de Agrotóxicos em Alimentos (PARA), que busca avaliar os níveis de resíduos de agrotóxicos em alimentos de origem vegetal.

De acordo com o relatório complementar de pesquisas desenvolvidas pelo PARA no ano de 2012, divulgado em 2014, foram analisadas 1.397 amostras, sendo que 347 (25\%) foram consideradas insatisfatórias, com pelo menos um dos fatores indesejáveis: Presença de agrotóxicos acima do Limite Máximo de Resíduo (LMR); Constatação de agrotóxicos não autorizados para determinadas culturas, ou as duas irregularidades. Das 229 amostras de abobrinha analisadas, 48\% (110 amostras) foram consideradas insatisfatórias, em decorrência, exclusivamente, do uso de carbofurano, agrotóxico não registrado para a cultura de abobrinha. ${ }^{1}$ No ano de 2016, foram divulgadas as análises dos anos de 2013 a 2015, sendo constatado que, das 216 amostras analisadas, em 168 foi identificada a presença de resíduos de agrotóxicos não autorizados para o uso na cultura de abobrinha, sendo encontrado dentre outros agrotóxicos o carbofurano. ${ }^{2}$

A mosca-branca (Bemisia spp) é uma praga que ataca as culturas de abobrinhas. De acordo com Schmalstig e Mcauslane, ${ }^{3}$ a praga prejudica a espécie por meio de sua alimentação, induzindo o prateamento das folhas. Summers e Stapleton ${ }^{4}$ ressaltam que essa

*e-mail: simone.goulart@ifg.edu.br praga ataca as culturas das espécies de C. pepo, C. moschata e C. máxima. Os carbamatos são muito eficientes para controle dessa praga em outras culturas, tais como a cultura do feijão, entretanto, não são autorizados para uso em culturas de abobrinha, e o uso ilegal do carbofurano nessa cultura é devido a essa eficiência no combate dessa praga. ${ }^{5}$

O carbofurano (2,3-dihidro-2,2dimethylbenzenofuran-7-ylmethylcarbamate) (Figura 1) pertence à classe química dos carbamatos, classificado pela Agência Nacional de Vigilância Sanitária (ANVISA) como inseticida, cupinicida, acaricida e nematicida. É classificado toxicologicamente como extremamente tóxico. É empregado no solo, principalmente em culturas de algodão, amendoim, arroz, banana, batata, café, cana-de-açúcar, cenoura, feijão, fumo, milho, repolho, tomate e trigo e nas sementes de algodão e arroz. ${ }^{6}$<smiles>CNC(=O)Oc1cccc2c1OC(C)(C)C2</smiles>

Figura 1. Estrutura química do carbofurano

Pela alta toxicidade dos carbamatos, muitos desses produtos químicos foram proibidos em diversos países, como na União Europeia, Estados Unidos e Canadá. ${ }^{7}$ No ano de 2015, a ANVISA abriu uma consulta pública para Reavaliar a Toxicidade do Ingrediente Ativo de Agrotóxico Carbofurano no Brasil, resultando em aprovação da proposta por $81,67 \%$ das respostas. ${ }^{8}$

O método QuEChERS é o recomendado pela ANVISA para determinação de carbofurano em abobrinha por cromatografia. ${ }^{9}$ Embora seja um método multirresíduo, o QuEChERS é um método bastante laborioso, se comparado a outros métodos de extração. 
Outro método que foi desenvolvido e que tem sido utilizado com sucesso para determinação de agrotóxicos em alimentos e demais matrizes por cromatografia é a Extração Sólido-Líquido com Partição em Baixa Temperatura (ESL/PBT). Este método já foi aplicado em várias matrizes como abacaxi, ${ }^{10}$ alface,${ }^{11}$ alho, ${ }^{12}$ batata,,${ }^{13,14}$ cenoura,${ }^{15}$ manteiga, ${ }^{16}$ milho, ${ }^{17}$ morango, ${ }^{18}$ solos ${ }^{19}$ e tomates. ${ }^{20}$ De acordo com Goulart et al.,$^{21}$ a técnica apresenta vantagens em relação às demais técnicas de extração, como praticidade, número reduzido de etapas e baixo consumo de solvente orgânicos.

O objetivo deste trabalho foi otimizar o método ESL/PBT para determinação de carbofurano em abobrinha por cromatografia líquida de alta eficiência com detector ultravioleta (HPLC-UV) e verificar a presença de carbofurano em amostras de abobrinhas comercializadas no município de Itumbiara - Goiás, Brasil.

\section{PARTE EXPERIMENTAL}

A otimização do ensaio experimental consistiu na utilização da técnica ESL-PBT, seguida de análise por HPLC-UV conforme metodologia proposta por Goulart et al. ${ }^{21}$

\section{Reagentes e soluções}

Os reagentes e solventes utilizados foram acetato de etila $(99,5 \%$ v/v - Mallinckrodt/HPLC) e acetonitrila (99,5\% v/v - J.T. Baker/ HPLC) e cloreto de sódio ( $99,5 \% \mathrm{~m} / \mathrm{m}$ - Synth).

A solução padrão estoque na concentração de $100,0 \mathrm{mg} \mathrm{L}^{-1}$ de carbofurano $(99,9 \% \mathrm{~m} / \mathrm{m}$ - Sigma Aldrich) foi preparada em acetonitrila e armazenada em freezer. A partir de diluição da solução estoque, foi preparada uma solução padrão de trabalho contendo o carbofurano na concentração de $5,0 \mathrm{mg} \mathrm{L} \mathrm{L}^{-1}$.

\section{Obtenção de amostras}

Para a otimização da técnica de extração sólido-líquido com partição em baixa temperatura (ESL/PBT) e análise por HPLC-UV foram utilizadas abobrinhas cultivadas sem adição de agrotóxicos (Branco) na zona rural do município de Itumbiara-GO, Brasil. Após coleta, as amostras de abobrinha foram refrigeradas e analisadas no Laboratório de Análise Química Instrumental do Instituto Federal de Goiás - Campus Itumbiara (IFG - Itumbiara), onde foram preparadas.

\section{Otimização da técnica ESL/PBT}

Amostras de abobrinhas isentas de agrotóxicos foram picadas e trituradas em mixer até total homogeneização. Em seguida, 1,0000 g de polpa da amostra foram colocadas em frascos de vidro transparente
(22 mL) e fortificadas com $2 \mu \mathrm{L}$ de solução-padrão de carbofurano a $5,0 \mathrm{mg} \mathrm{L}^{-1} \mathrm{e}$ homogeneizadas. Às amostras fortificadas (na presença e ausência de adição de sal) foram acrescentadas água ( $2 \mathrm{~mL})$ e solventes extrator na proporção (1:2). O sistema foi agitado em vórtex e as amostras foram levadas ao freezer a $-20{ }^{\circ} \mathrm{C}$ por no mínimo 3 horas para separação das fases. Após este intervalo, foi retirado 1 $\mathrm{mL}$ da fase orgânica e colocado em vial para análise cromatográfica.

Para a otimização do método foi empregado um planejamento fatorial completo, $2^{3}$, para avaliação do comportamento simultâneo de três fatores: tempo de agitação em vórtex (30 ou 60 s), adição de sal (sem ou com $1,5 \%(\mathrm{~m} / \mathrm{m})$ de $\mathrm{NaCl})$ e solvente extrator (acetonitrila- ACN ou acetonitrila:acetato de etila - ACN/ACE 81,25:18,75). Os três fatores foram analisados em dois níveis e as análises foram realizadas em triplicatas. As melhores condições do planejamento foram avaliadas em função das porcentagens de extração obtidas em cada ensaio. A precisão do método otimizado foi determinada por meio do coeficiente de variação $(\mathrm{CV})$ para nove extrações idênticas de brancos fortificados a $5 \mathrm{mg} \mathrm{L}^{-1}$.

Esses dados foram tratados empregando o programa Excel $^{\circledR}$. Os dados do planejamento são apresentados na Tabela 1 .

\section{Condições cromatográficas}

As seguintes condições cromatográficas foram ajustadas para determinação de carbofurano por HPLC: Coluna Kinetex $5 \mu \mathrm{m}$ EVO C 18 - 150 X 4,6 mm; fase móvel (modo isocrático): acetonitrila:água purificada 35:65 v:v; vazão da fase móvel: $0,8 \mathrm{~mL} \mathrm{~min}^{-1}$; temperatura da coluna: $35^{\circ} \mathrm{C}$; volume de injeção $20 \mu \mathrm{L}$ e pressão: $67 \mathrm{kgf}$. Detector ultravioleta (UV) $(\lambda: 195 \mathrm{~nm})$.

A quantificação do carbofurano nas amostras de abobrinha foi realizada pelo método da padronização externa utilizando padrão analítico de carbofurano no extrato do branco da matriz. A partir de diluições da solução de carbofurano a $5 \mathrm{mg} \mathrm{L}^{-1}$ foram obtidas as soluções padrão nas concentrações de $0,1 \mathrm{mg} \mathrm{L}^{-1}, 0,5 \mathrm{mg} \mathrm{L}^{-1}, 1 \mathrm{mg}$ $\mathrm{L}^{-1}, 2 \mathrm{mg} \mathrm{L}{ }^{-1}, 3 \mathrm{mg} \mathrm{L}^{-1}, 4 \mathrm{mg} \mathrm{L}^{-1}$ e $5 \mathrm{mg} \mathrm{L}^{-1}$.

\section{Limite de detecção e limite de quantificação}

Os limites de detecção (LOD) e os limites de quantificação (LOQ) do método proposto foram determinados pela fortificação dos extratos de abobrinha nas concentrações mais baixas aceitáveis. LOD e LOQ foram considerados como três e dez vezes, respectivamente, o sinal de ruído de linha de base obtido para as amostras livres de carbofurano (em branco). Os extratos de abobrinha foram obtidos submetendo-se o branco (abobrinha livre de carbofurano) ao método ESL-PBT otimizado nesse trabalho. Foram realizadas várias injeções dos extratos, fortificados em concentrações decrescentes. ${ }^{22}$

Tabela 1. Planejamento fatorial $2^{3}$, em triplicata, realizado para estabelecer melhores condições para extração de carbofurano de amostras de abobrinha

\begin{tabular}{|c|c|c|c|c|c|c|c|}
\hline \multirow{2}{*}{ Ensaios } & \multirow{2}{*}{ Replicatas } & \multicolumn{3}{|c|}{ Fatores Codificados } & \multicolumn{3}{|c|}{ Fatores Originais } \\
\hline & & $\mathrm{F}(1)$ & $\mathrm{F}(2)$ & $\mathrm{F}(3)$ & (1) Tempo Agitação (S) & (2) Adição $\mathrm{NaCl}(\% \mathrm{~m} / \mathrm{v})$ & (3) Solução Extratora \\
\hline 1 & 1,2 e 3 & - & - & - & 30 & 0 & $\mathrm{ACN}$ \\
\hline 2 & 4,5 e 6 & + & - & - & 60 & 0 & $\mathrm{ACN}$ \\
\hline 3 & 7,8 e 9 & - & + & - & 30 & 1,5 & $\mathrm{ACN}$ \\
\hline 4 & 10,11 e 12 & + & + & - & 60 & 1,5 & $\mathrm{ACN}$ \\
\hline 5 & 13,14 e 15 & - & - & + & 30 & 0 & $\mathrm{ACN} / \mathrm{ACE}$ \\
\hline 6 & 16,17 e 18 & + & - & + & 60 & 0 & $\mathrm{ACN} / \mathrm{ACE}$ \\
\hline 7 & 19,20 e 21 & - & + & + & 30 & 1,5 & ACN/ACE \\
\hline 8 & 22,23 e 24 & + & + & + & 60 & 1,5 & $\mathrm{ACN} / \mathrm{ACE}$ \\
\hline
\end{tabular}




\section{Aplicação do método otimizado em amostras de abobrinha no comércio}

Após a otimização do método, este foi aplicado em 5 (cinco) amostras de abobrinha coletadas em cinco estabelecimentos comerciais da cidade de Itumbiara-GO, Brasil. As amostras foram refrigeradas e analisadas após $24 \mathrm{~h}$.

\section{RESULTADOS E DISCUSSÃO}

\section{Curva analítica}

Foram realizadas injeções de soluções padrão de carbofurano no extrato do branco da matriz abobrinha, com o objetivo de verificar a resposta do detector frente às diferentes concentrações do analito (0,1 $\mathrm{mg} \mathrm{L}^{-1}$ a 5,0 $\left.\mathrm{mg} \mathrm{L}^{-1}\right)$. A partir dos dados obtidos foi construída a curva analítica $y=308605 \mathrm{x}-10475$ com coeficiente de correlação igual a 0,9975 .

Como a medição nos ensaios químicos é indireta, a transformação da medida do equipamento na unidade de interesse do mensurando é feita utilizando uma comparação direta de uma série de soluções padrão de concentrações conhecidas contra os valores medidos, seguida de transformações matemáticas até a obtenção do resultado final. ${ }^{23}$

Observa-se que a equação da reta apresentou coeficiente de correlação igual a 0,9975 , considerada uma Correlação Altíssima $(0,91$ $<\mathrm{r}<0,99)$ de acordo com Brito et al. ${ }^{24}$ que também afirmam que este parâmetro se refere à capacidade do método de gerar resultados linearmente proporcionais à concentração do analito, enquadrados em faixa analítica especificada.

\section{Análise cromatográfica}

As condições cromatográficas otimizadas para análise de carbofurano em abobrinha possibilitaram a identificação do pesticida no tempo de retenção de 5,34 min, conforme apresentado no cromatograma da Figura 2.

\section{Otimização da técnica ESL/PBT}

No processo de otimização do método foi empregado um planejamento fatorial completo, $2^{3}$, para avaliação do comportamento simultâneo de três fatores: tempo de agitação em vórtex (30 ou $60 \mathrm{~s}$ ), adição de sal (sem ou com 1,5\% (m/m) de $\mathrm{NaCl})$ e solvente extrator (acetonitrila- ACN ou acetonitrila:acetato de etila - ACN/ ACE 81,25:18,75). Os resultados das combinações dos fatores foram registrados para análise e interpretação em planilhas eletrônicas, conforme Teófilo e Ferreira. ${ }^{25}$ A escolha das melhores condições para aplicação da técnica ESL/PBT favorece melhores resultados, levando a maior frequência analítica (agilidade nas análises por unidade de tempo) e menor custo. Os resultados da análise estatística são apresentados na Tabela 2.

Tabela 2. Porcentagens de recuperação média, efeitos e interação entre os fatores, erro experimental e p-Valor

\begin{tabular}{lrlc}
\hline Fatores & Efeitos & Erros & p-valor \\
\hline \%Média de recuperação & $75,88 \pm$ & 0,87 & $7,9.10^{-23}$ \\
(1) Tempo de agitação & $0,23 \pm$ & 1,74 & 0,89 \\
(2) Adição NaCl & $3,06 \pm$ & 1,74 & 0,09 \\
(3) Solução Extratora & $1,58 \pm$ & 1,74 & 0,37 \\
Interação 1 x 2 & $-0,76 \pm 1,74$ & 0,66 \\
Interação 1 x 3 & $-0,41 \pm 1,74$ & 0,81 \\
Interação 2 x 3 & $2,97 \pm 1,74$ & 0,10 \\
Interação 1 x 2 x 3 & $-2,06 \pm 1,74$ & 0,25 \\
\hline
\end{tabular}

Para verificação da significância dos dados apresentados na Tabela 2, foi aplicado o teste t de t-student ao nível de $95 \%$ de confiança e 5\% significância. Para 8 graus de liberdade com $95 \%$ de probabilidade o valor t correspondente é 2,306, dessa forma serão significativos os fatores com valores excedentes a $(0+2,306$ x 1,74) $=4,01$, análogo a dizer que serão significativos todos os efeitos cujo valor de $\mathrm{p}<0,05 .{ }^{25}$ Verifica-se na Tabela 2 que estatisticamente, nos níveis estabelecidos, os fatores não são significativos ao ponto de interferir no aumento ou diminuição das porcentagens de recuperação do carbofurano na matriz abobrinha. Entretanto, a fim de selecionar o melhor ensaio para determinação e quantificação de carbofurano na matriz investigada, é de fundamental importância entender a atuação de cada fator no processo.

Observando-se o processo de agitação das amostras, foi verificado que elevando o tempo de agitação de $30 \mathrm{~s}$ para $60 \mathrm{~s}$, o percentual de recuperação aumentou em média $0,235 \%$. Esta etapa é de fundamental importância no processo analítico por representar o momento de maior interação entre o solvente extrator e o analito, proporcionando melhor precisão, praticidade, frequência analítica e alto rendimento. ${ }^{26}$ Por esse motivo foi escolhido o tempo de agitação de $60 \mathrm{~s}$.

A adição de $\mathrm{NaCl}$ ao sistema foi o fator que mais influenciou no aumento das porcentagens de recuperação. Com o aumento da força iônica do sistema, observou-se um aumento de 3,06\% na eficiência da extração. Geralmente, a introdução de sais, como $\mathrm{NaCl}$, à fase aquosa do sistema permite obter maiores percentuais de recuperação

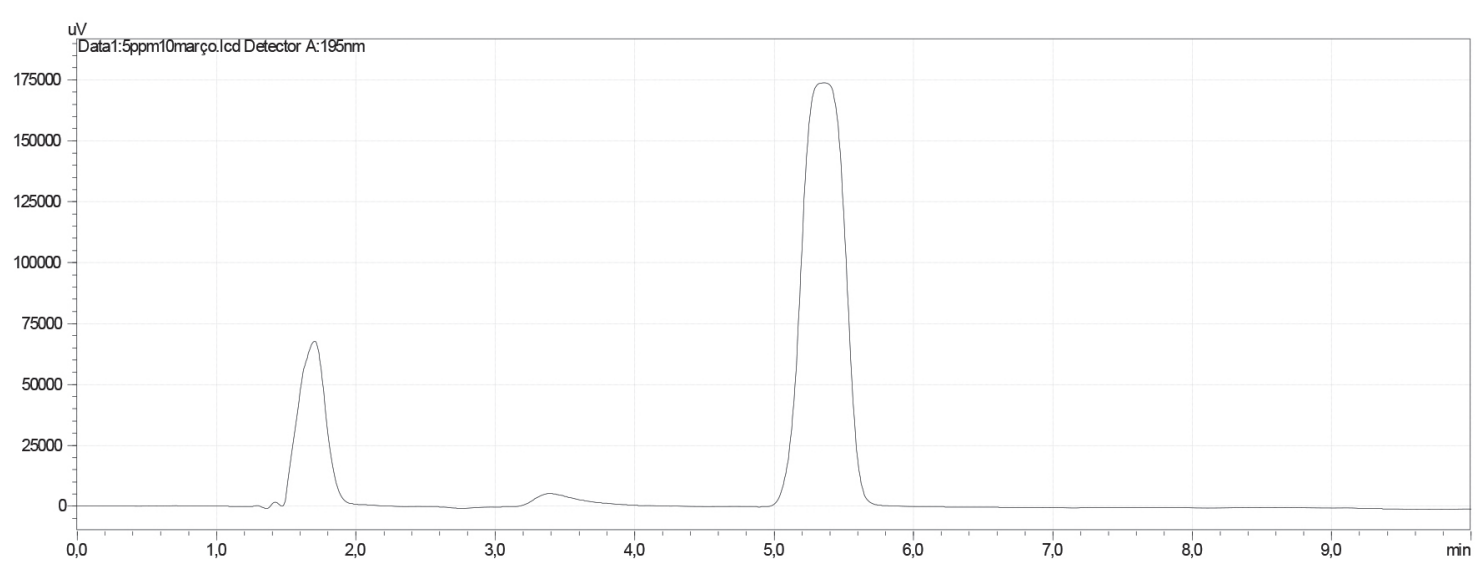

Figura 2. Cromatograma de uma solução padrão a 5,0 $\mathrm{mg} \mathrm{L}^{-1}$ de carbofuran $\left(t_{R}=5,34 \mathrm{~min}\right.$ ) em acetonitrila. 
para compostos orgânicos, pois diminui a solubilidade destes na fase aquosa, favorecendo a transferência destes para a fase orgânica. ${ }^{26}$

Com relação à solução extratora, observou-se que nos ensaios utilizando apenas acetonitrila como solvente extrator, a recuperação média foi de $73,1 \%$, enquanto que, empregando a mistura acetonitrila: acetato de etila $(81,25: 18,75)$ obteve-se média de recuperação de $76,7 \%$. A recuperação média de todos os ensaios foi $75,88 \%$. A precisão do método foi determinada através do coeficiente de variação $(\mathrm{CV})$ para nove extrações idênticas de brancos fortificados a $5 \mathrm{mg} \mathrm{L}^{-1}$ e os resultados foram $73,7 \%$ com coeficiente de variação de 5,3\%. Resultados semelhantes foram obtidos na determinação de agrotóxicos em tomates realizada por Pinho et al. ${ }^{20}$ quando empregaram a combinação de dois solventes. A mesma técnica foi aplicada por Costa et al. ${ }^{11}$ para determinação de agrotóxicos em amostras de alface, obtendo percentuais de recuperação entre 72,3\% e 103,2\%. Atualmente a técnica ESL/PBT foi aplicada na análise de bifenilas policloradas em lodo de esgoto e os percentuais de extração foram superiores a $82 \% .{ }^{27}$

Além da eficiência de extração e compatibilidade com a técnica cromatográfica, outros fatores devem ser avaliados para escolha do melhor solvente extrator, entre eles a viabilidade econômica e o descarte dos resíduos dos solventes orgânicos.

Como as médias de recuperação estatisticamente não diferem entre si, considerando que a acetonitrila é miscível em água e apresenta baixo ponto de fusão $\left(-45^{\circ} \mathrm{C}\right)$, propriedades essenciais para se empregar a ESL/PBT, este foi o solvente extrator escolhido. ${ }^{28}$

Assim, o ensaio 4 (repetições 10, 11 e 12) com tempo de agitação em vortex - $60 \mathrm{~s}$; adição de $\mathrm{NaCl}$ e $\mathrm{ACN}$ como solvente extrator foram escolhidos para dar continuidade ao trabalho, em virtude da viabilidade prática e econômica do experimento.

Na Figura 3 está apresentado um cromatograma de um extrato do branco da abobrinha e cromatogramas obtidos de amostras de abobrinha livres de carbofurano, fortificadas e submetidas ao Ensaio 4. Observa-se que o tempo de retenção do carbofurano está entre 5 e 6 minutos. $\mathrm{O}$ extrato do branco não apresenta picos no tempo de retenção dos carbofurano, o que permite afirmar que o método proposto apresenta seletividade..$^{29}$

\section{Limite de detecção e quantificação}

Os limites de detecção e quantificação do método proposto foram $0,04 \mathrm{mg} \mathrm{kg}^{-1}$ e $0,13 \mathrm{mg} \mathrm{kg}^{-1}$, respectivamente. Resultados semelhantes foram obtidos por Goulart et al. ${ }^{24}$ na análise de carbofurano em matrizes de bebidas de interesse forense. Este método é conhecido como método do sinal-rúído. Na determinação da relação sinal-ruído, faz-se a comparação entre a medição dos sinais de amostras em baixas concentrações conhecidas do composto de interesse na matriz e um branco (matriz isenta do composto de interesse) destas amostras. ${ }^{22}$

O carbofurano não é registrado na ANVISA para utilização em culturas de abobrinha, embora no último PARA tenha se verificado a presença desse agrotóxico nessa cultura. É importante ressaltar que estudos recentes realizados no município de Itumbiara-GO, alertam sobre a gama de agrotóxicos utilizados nos cultivos da região, em especial para a cana-de-açúcar e hortaliças. Em uma pesquisa realizada por Rodrigues et al. ${ }^{30}$ na cidade de Itumbiara-GO, foi constatado que $100 \%$ dos produtores rurais entrevistados utilizam algum tipo de agrotóxico, incluindo os carbamatos, como o carbofurano. No que se refere ao fácil acesso destes produtos identificou-se o relato de suicídio devido ao uso do agrotóxico Furadan ${ }^{\circledR}$, que apresenta o carbofurano como princípio ativo, além da verificação de problemas ambientais relacionados ao descarte inadequado de embalagens e mortes de animais decorrentes de aplicações aéreas de agrotóxicos. Do ponto de vista da segurança alimentar, a ausência de resíduos de carbofurano nas amostras analisadas é de suma importância, porém a quantidade de agrotóxicos utilizados na região é grande e os tipos de vegetais comercializados são inúmeros. Diante deste cenário torna-se evidente a necessidade de manutenção de pesquisas sobre resíduos de agrotóxicos em alimentos e os impactos ambientais decorrentes do uso indiscriminado nas culturas da região.

\section{Aplicação do método de ESL/PBT-HPLC/UV em amostras de abobrinhas comerciais}

As amostras de abobrinha comercializadas no munícipio de Itumbiara-GO, coletadas e processadas conforme descrito

a)

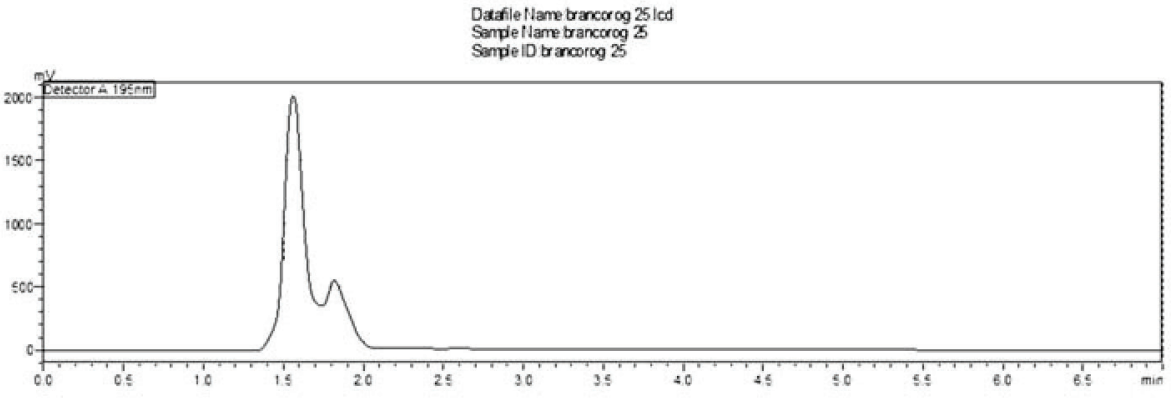

b)

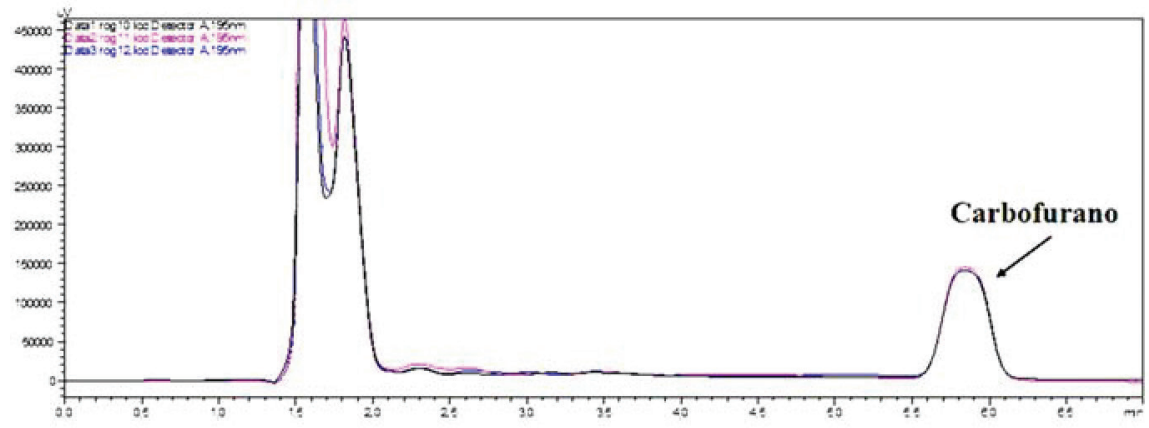

Figura 3. (a) Cromatograma de um extrato obtido de abobrinha isenta dos princípios ativos e (b) Cromatograma de extratos de abobrinha contendo o carbofurano a $5,0 \mathrm{mg} \mathrm{L}^{-1}$. 


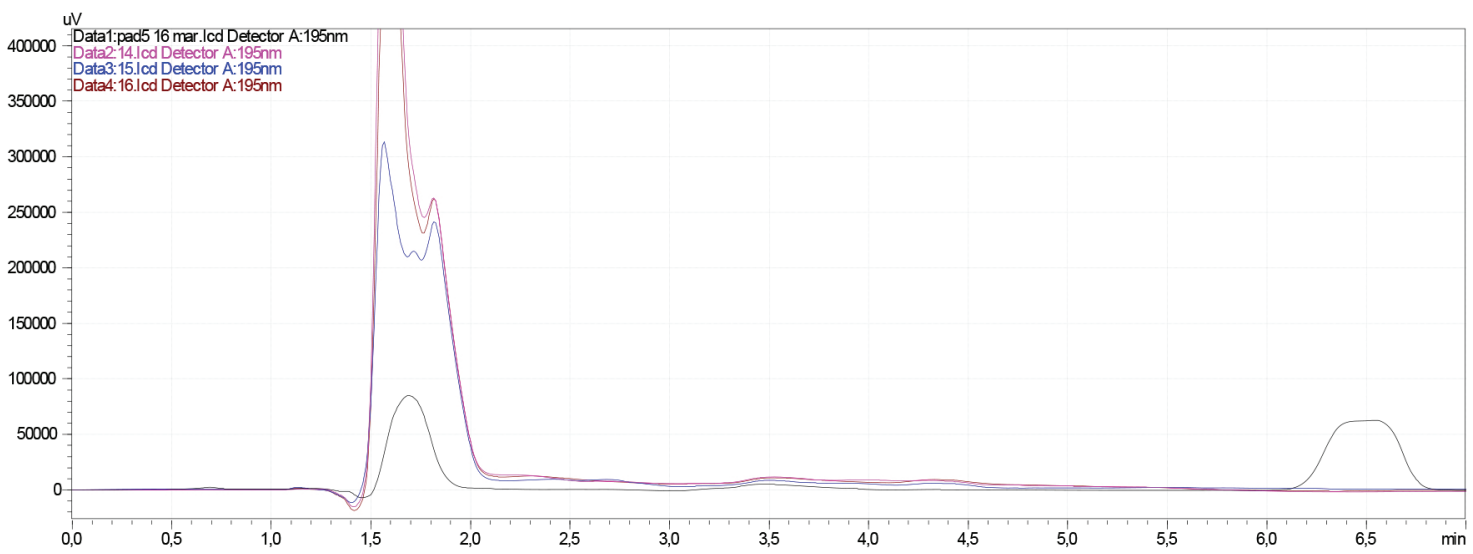

Figura 4. Cromatograma de extratos obtidos de matrizes reais de abobrinha (amostra 1) (-) (—) (-) replicatas comparadas ao padrão do carbofurano $5,0 \mathrm{mg} \mathrm{L}^{-1}($ -

anteriormente foram submetidas, em triplicata, ao método otimizado de ESL/PBT-HPLC/UV.

As condições do ensaio 4 (quatro) do planejamento (tempo de agitação em vortex - $60 \mathrm{~s}$; adição de $\mathrm{NaCl}$ e ACN como solvente extrator) foram aplicados nas amostras reais para determinação de carbofurano. À 1,0000 g de polpa da amostra comercial de abobrinha, colocada em frascos de vidro transparente $(22 \mathrm{~mL})$ foi adicionado o sal $\mathrm{NaCl}(1,5 \% \mathrm{~m} / \mathrm{m})$. Após, foram acrescentados água $(2 \mathrm{~mL})$ e acetonitrila $(4 \mathrm{~mL})$ na proporção $(1: 2)$. O sistema foi agitado em vórtex por $60 \mathrm{~s}$ e as amostras foram levadas ao freezer a $-20{ }^{\circ} \mathrm{C}$ por 3 horas para separação das fases. Após este intervalo, foi retirado 1 $\mathrm{mL}$ da fase orgânica e colocado em vial para análise cromatográfica.

Não foi evidenciada a presença de carbofurano dentro da faixa de detecção do aparelho em todas as cinco amostras avaliadas, o cromatograma das análises em triplicata, da amostra de abobrinha 1 é apresentado na Figura 4. Os cromatogramas das amostras 2 a 5 foram semelhantes ao cromatograma da amostra 1.

O carbofurano não é autorizado pela ANVISA para aplicação em culturas de abobrinha. Para as culturas de cenoura, repolho e tomate os valores de LMR são $0,5 \mathrm{mg} \mathrm{kg}^{-1}, 1,0 \mathrm{mg} \mathrm{kg}^{-1} \mathrm{e} 0,1 \mathrm{mg} \mathrm{kg}^{-1}$, respectivamente. ${ }^{6}$

\section{CONCLUSÃO}

A técnica ESL/PBT aplicada na determinação de carbofurano em matrizes de abobrinha é seletiva, simples e eficaz, consumindo pequenas quantidades de solventes e amostras, com porcentagem média de recuperação de 75,88\%. Além disso, não são necessárias etapas posteriores de clean-up do extrato. Após a PBT os extratos são analisados diretamente por HPLC-UV.

As análises de matrizes reais de abobrinhas comercializadas no município de Itumbiara-GO utilizando a técnica ESL-PBT não apresentaram resíduos do agrotóxico carbofurano.

\section{AGRADECIMENTOS}

Ao Ministério da Educação (MEC) e a Fundação Nacional de Desenvolvimento da Educação (FNDE) pelo o apoio financeiro concedido ao Programa de Educação Tutorial (PET) do Instituto Federal de Goiás - Campus Itumbiara.

\section{REFERÊNCIAS}

1. Agência Nacional de Vigilância Sanitária (ANVISA). Disponível em http://portal.anvisa.gov.br/documents/111215/117818/Relat\%25C3\%
25B3rio\%2BPARA\%2B2012\%2B2\%25C2\%25AA\%2BEtapa\%2B\%2B17_10_14-Final.pdf/3bc220f9-8475-44ad-9d96-cbbc988e28fa, acessado em outubro de 2017.

2. Agência Nacional de Vigilância Sanitária (ANVISA). Disponível em http://portal.anvisa.gov.br/documents/111215/0/ Relat\%C3\%B3rio+PARA+2013-2015_VERS\%C3\%83O-FINAL. pdf/494cd7c5-5408-4e6a-b0e5-5098cbf759f8, acessado em outubro de 2017.

3. Schmalstig, J. C.; Mcauslane, H. J.; J. Am. Soc. Hortic. Sci. 2001, 126, 544.

4. Summers, C. G.; Stapleton, J. J.; Crop Prot. 2002, 21, 921.

5. Scarpellini, J. R.; Ramiro, Z. A.; Lara, R. I. R.; Santos, J. C. C.; Arq. Inst. Biol. 2002, 69, 23.

6. Agência Nacional de Vigilância Sanitária (ANVISA). Disponível em http://portal.anvisa.gov.br/documents/111215/117782/C06\%2B\%25 E2\%2580\%2593\%2BCarbofurano.pdf/84d57401-49cc-47fd-9db62be75c116548, acessado em outubro de 2017.

7. Fundação Nacional de Saúde (FUNASA). Disponível em: http://bvsms. saude.gov.br/bvs/publicacoes/funasa/controle_vetores.pdf, acessado em outubro de 2017.

8. http://portal.anvisa.gov.br/documents/33880/2541557/CP\%2B1142015\%2B-\%2BNota\%2Btecnica.pdf/b2b515d7-8e0e-4884-b7a9525442 b1bacb, acessado em outubro de 2017.

9. Prestes, O. D.; Adaime, M. B.; Zanella, R.; Sci. Chromatogr. 2011, 3, 51.

10. Morais, E. H. C.; Rodrigues, A. A. Z.; Queiroz, M. E. L. R.; Neves, A. A.; Morais, P. H. D.; Food Control. 2014, 42, 9.

11. Costa, A. I. G.; Queiroz, M. E. L. R.; Neves, A. A.; Sousa, F. A.; Zambolim, L.; Food Chem. 2015, 181, 64.

12. Moreno, C. M.; Stadler, T.; Silva, A. A.; Barbosa, L. C. A.; Queiroz, M. E. L. R.; Talanta 2012, 89, 369.

13. Rigueira, L. M. B.; Ribeiro, L. K.; Queiroz, M. E. L. R.; Neves, A. A.; Zambolim, L.; Oliveira, R. M.; J. Braz. Chem. Soc. 2013, 24, 2042.

14. Dardengo, R. P.; Goulart, S. M.; Neves, A. A.; Zambolim, L.; Queiroz, M. E. L. R.; Braz. J. Anal. Chem. 2011, 3, 136.

15. Araújo, E. A.; lara, M. C. R.; Reis, M. R.; Viriato, R. L. S.; Rocha, R. A. R.; Gonçalves, R. G. L.; Heleno, F. F.; Queiroz, M. E. L. R.; Tronto, J.; Pinto, F. G.; Food Analytical Methods 2016, 9, 1344.

16. Marthe, D. B.; Bittencourt, L. M.; Queiroz, M. E. L. R.; Neves, A. A.; Quim. Nova 2010, 33, 1389.

17. Freitas, R. S.; Queiroz, M. E. L. R.; Faroni, L. R. A.; Heleno, F. F.; Moura, V. V.; Quim. Nova 2014, 37, 238.

18. Heleno, F. F.; Queiroz, M. E. L. R.; Neves, A. A.; Oliveira, A. F.; Quim. Nova. 2014, 37, 153.

19. Assis, E. C.; Silva, A. A.; Barbosa, L. C.; Queiroz, M. E. L. R.; 
D'Antonino, L.; Gonçalves, V. A.; Planta Daninha 2011, 29, 683.

20. Pinho, G. P.; Neves, A. A.; Queiroz, M. E. L. R.; Silverio, F. O.; Food Chem. 2010, 121, 251.

21. Goulart, S. M.; Alves, R. D.; Neves, A. A.; Queiroz, J. H.; Assis, T. C.; Queiroz, M. E. L.; Anal. Chim. Acta 2010, 671, 41.

22. Ribani, M.; Bottoli, C. B. G.; Collins, C. H.; Jardim, I. C. S. F.; Melo, L. F. C.; Quim. Nova 2004, 27, 771.

23. Bahia filho, O.; Prada, P. R.; Meneghesso, C.; Lanças, F. M.; Sci. Chromatogr. 2011, 3, 251.

24. Brito, N. M.; Amarante Junior, O. P.; Polese, L.; Ribeiro, M. L.; Pesticidas: Revista de Ecotoxicologia e Meio Ambiente 2003, 13, 129.
25. Teófilo, R. F.; Ferreira, M. M. C.; Quim. Nova 2006, 29, 338.

26. Prestes, O. D.; Friggi, C. A.; Adaime, M. B.; Zanella, R.; Quim. Nova 2009, 32, 1620.

27. Maia, M. R.; Arcanjo, A. L. P.; Pinho, G. P.; Silvério, F. O.; J. Braz. Chem. Soc. 2017, 28, 179.

28. Maštovská. K.; Lehotay, S. J.; J. Chromatogr. A 2004, 1040, 259.

29. European Commission. SANTE/11945/2015. Disponível em: https:// ec.europa.eu/food/sites/food/files/plant/docs/pesticides_mrl_guidelines_ wrkdoc_11945.pdf, acessado outubro de 2017.

30. Rodrigues, V. C.; Goulart, S. M.; Santos, J. P. V.; Ribeiro Filho, H. A.; Castro, L. M.; Bernadeli, A. A.; Gomes, T. C. F.; Sinergia 2016, 17, 56. 